\title{
A simple and inexpensive haemozoin-based colorimetric method to evaluate anti-malarial drug activity
}

Tran Thanh Men ${ }^{1,2}$, Nguyen Tien Huy ${ }^{3^{*}}$, Dai Thi Xuan Trang ${ }^{1}$, Mohammed Nasir Shuaibu ${ }^{3}$, Kenji Hirayama $a^{3,4}$ and Kaeko Kamei ${ }^{2^{*}}$

\begin{abstract}
Background: The spread of drug resistance in malaria parasites and the limited number of effective drugs for treatment indicates the need for new anti-malarial compounds. Current assays evaluating drugs against Plasmodium falciparum require expensive materials and equipment, thus limiting the search for new drugs, particularly in developing countries. This study describes an inexpensive procedure that is based on the advantage of a positive correlation between the haemozoin level of infected erythrocytes and parasite load.

Methods: The relationship between parasitaemia and the haemozoin level of infected erythrocytes was investigated after converting haemozoin into monomeric haem. The $50 \%$ inhibitory concentration $\left(\mathrm{IC}_{50}\right)$ values of chloroquine, quinine, artemisinin, quinidine and clotrimazole against $P$. falciparum $\mathrm{K} 1$ and $9 \mathrm{~A}$ strains were determined using the novel assay method.

Results: The haemozoin of parasites was extracted and converted into monomeric haem, allowing the use of a colorimeter to efficiently and rapidly measure the growth of the parasites. There was a strong and direct linear relationship between the absorbance of haem converted from haemozoin and the percentage of the parasite $\left(R^{2}=0.9929\right)$. Furthermore, the $I C_{50}$ values of drugs were within the range of the values previously reported.

Conclusion: The haemozoin-based colorimetric assay can be considered as an alternative, simple, robust, inexpensive and convenient method, making it applicable in developing countries.
\end{abstract}

Keywords: Anti-malarial, Assay, Haemozoin, Malaria

\section{Background}

Malaria is more than just a problem for tropical countries, it also is a major global public health concern. Annually, there are approximately 300 million new malaria infections and millions of deaths worldwide due to malaria $[1,2]$. Because a vaccine for malaria is not available, chemotherapy is the main treatment. However, the rapid spread of resistance to current quinoline anti-malarials has made malaria a major global and important problem. In addition, artemisinin, from a Chinese herb (Qinghaosu) that has been used in the treatment of fevers for

\footnotetext{
*Correspondence: tienhuy@nagasaki-u.ac.jp; kame@kit.ac.jp

2Department of Biomolecular Engineering, Kyoto Institute of Technology,

Matsugasaki, Sakyo-ku, Kyoto 606-8585, Japan

${ }^{3}$ Department of Immunogenetics, Institute of Tropical Medicine (NEKKEN)

Nagasaki University, Nagasaki, Japan

Full list of author information is available at the end of the article
}

more than a thousand years, is now considered an essential component of artemisinin-based combination therapy against drug-resistant malaria [3,4]. However, the malaria parasites have recently been found to be resistant to artemisinin [5-7]. The alarming spread of drug resistance and the limited number of effective drugs for treatment indicates how important it is to find new antimalarial compounds.

For decades, the anti-malarial activity of a drug has been measured in vitro by quantifying the uptake of radioactive substrates by a parasite as a measure of growth and viability in the presence of the test drug $[8,9]$. Although several in vitro methods exist, the ${ }^{3} \mathrm{H}-$ hypoxanthine method [8] is a popular test for novel anti-malarial drugs, but it is labelled with radiation that presents a potential risk to safety, and it relies on 
relatively expensive radio-isotopes and includes multistep procedures that become increasingly problematic and impractical when the incidence of testing is increased. The other methods, including the PicoGreen $[10,11]$ and the SYBR Green I $[12,13]$ methods, are also considered to be expensive approaches regarding equipment and chemicals. In addition, there are other methods that are based on enzymatic reaction and/or antibodies that specifically detect the presence of histidine-rich protein II or parasite lactate dehydrogenase [14-16]. However, these methods involve multiple complex steps that are too expensive for developing countries, which makes them ill suited for screening potential anti-plasmodial drugs.

During the development and proliferation stage in host erythrocytes, the malaria parasites degrade haemoglobin for use as a major source of amino acids. This is accompanied by the release of free haem. With haem as a prosthetic group of haemoglobin, the iron is in the ferrous state, but free haem loses one electron and assumes the ferric state. This ferric haem could be oxidatively active and toxic to both the host cells and malaria parasites, and can even cause parasite death. Moreover, due to the absence of haem oxygenase, the parasite is unable to cleave haem into an open-chain free haem, which is necessary for cellular excretion [17]. To protect itself, it is necessary for the parasite to convert haem to non-toxic metabolites. Principally, the parasite detoxifies free haem via neutralization with histidine-rich protein $2[18,19]$ and degradation with reduced glutathione [20,21], but mostly with crystallization into haemozoin, which is a water-insoluble malarial pigment produced in the food vacuole $[19,22]$. Therefore, a simple and inexpensive in vitro assay was developed based on the colorimetric quantification of haemozoin in infected red blood cells to evaluate the anti-malarial activity of drugs.

\section{Methods \\ Materials}

Chloroquine diphosphate, quinine sulphate, primaquine, clotrimazole, haemin chloride (haem), RPMI 1640 medium, hypoxanthine, and gentamycin were purchased from Sigma Aldrich Chemical Company (Tokyo, Japan). Albumax II (Gibco), and the other chemicals used in the present study were of a high grade. Plasmodium falciparum K1 (chloroquine resistant) and 3D7-9A (chloroquine susceptible) strains [23] were provided from Dr Osamu Kaneko and Dr Shusuke Nakazawa, respectively, from the Institute of Tropical Medicine, Nagasaki University, Japan.

\section{Plasmodium cultivation}

Plasmodium falciparum K1 and 9A strains were maintained in vitro with continuous culture according to a previously described method with a slight modification [24]. The culture medium consisted of RPMI 1640 supplemented with $0.025 \mathrm{mg} / \mathrm{ml}$ gentamicin, $0.01 \mathrm{mM}$ hypoxanthine, $23.8 \mathrm{mM} \mathrm{NaHCO}, 11 \mathrm{mM}$ glucose and $0.5 \%$ albumax II, and adjusted to a $\mathrm{pH}$ of 7.3 to 7.4 . The parasite was cultured and maintained in a tissue culture flask with complete culture medium containing 5\% human erythrocytes. The parasite density was maintained at about 1.5\% parasitaemia under an atmosphere of an AnaeroPack sachet (Mitsubishi Gas Chemical Company Inc, Tokyo, Japan) to create $20 \% \mathrm{CO}_{2}$ and remove $\mathrm{O}_{2}(<0.1 \%) 37^{\circ} \mathrm{C}$ [25]. Every two days, infected erythrocytes were transferred into fresh medium containing $5 \%$ human erythrocyte. The level of parasitaemia was determined by light microscopy on a Giemsa-stained thin blood smear, and parasitized erythrocytes were diluted when parasitaemia was higher than $5 \%$ in erythrocytes contained at $5 \%$ in culture medium, in order to lower parasitaemia and allow continuous growth. Parasite culture was diluted with fresh uninfected erythrocytes and culture medium to achieve a starting parasitaemia of $2 \%$ and a haematocrit of $5 \%$. This final parasite culture was immediately used for anti-malarial assay.

\section{The relationship between parasitaemia and haemozoin level}

A culture of the P. falciparum K1 strain was serially diluted with uninfected erythrocytes in complete medium to yield a haematocrit of $5 \%$ and parasitaemia ranging from 0 to $10 \%$. The serial culture containing $200 \mu \mathrm{l}$ was prepared independently in triplicate in microtubes, followed by the addition of $800 \mu \mathrm{l}$ of $2.5 \%$ sodium dodecyl sulphate in $0.1 \mathrm{M}$ sodium bicarbonate $\mathrm{pH} 8.8$, then the samples were mixed at room temperature for $15 \mathrm{~min}$. After centrifugation at 13,000 rpm for $10 \mathrm{~min}$, the supernatant was removed. The pellet was washed twice with $800 \mu \mathrm{l}$ of $2.5 \%$ sodium dodecyl sulphate in $0.1 \mathrm{M}$ sodium bicarbonate $(\mathrm{pH} 8.8)$, then $200 \mu \mathrm{l}$ of $5 \%$ sodium dodecyl sulphate was added to $50 \mathrm{mM} \mathrm{NaOH}$ to convert the haemozoin into haem. After incubation at room temperature for $30 \mathrm{~min}$, the sample $(200 \mu \mathrm{l})$ was transferred to a 96well microplate and scanned at $405 / 750 \mathrm{~nm}\left(\mathrm{~A}_{405} \mathrm{~nm}\right.$ minus $A_{750} \mathrm{~nm}$ ) using an IMark microplate reader (BioRad). After the background absorbance of haemozoin was purified of uninfected erythrocytes (5\% haematocrit) then subtracted, the amount of haemozoin in the infected erythrocytes was presented as the absorbance at $405 / 750 \mathrm{~nm}$ and then plotted against parasitaemia.

Evaluating the anti-malarial activity of drugs using the haemozoin-based spectrophotometric method

The P. falciparum K1 and 9A strains were used to evaluate the anti-malarial activity of quinine, chloroquine, 
pyrimethamine, artemisinin and clotrimazole by using the haemozoin-based spectrophotometric method. Stocks of drugs were prepared in dimethyl sulphoxide or phosphate buffer saline (for chloroquine) and were then serially diluted with complete culture medium. To each well of a microplate, $10 \mu \mathrm{l}$ of serially diluted drug solution was added into $200 \mu \mathrm{l}$ of final asynchronous parasite culture. Dimethyl sulphoxide or phosphate buffer saline were also tested by adding a similar amount to control wells. The microplates were cultured $72 \mathrm{hr}$ under the conditions described above. The haemozoin of infected erythrocytes was extracted, purified, and quantified, as described above. The $50 \%$ inhibitory concentration $\left(\mathrm{IC}_{50}\right)$ value was calculated by non-linear fitting of the absorbance at $405 / 750 \mathrm{~nm}$ against the logarithm of the drug concentration using the GraphPad Prism 5 (GraphPad Software, Inc., San Diego, CA, USA). Sigmoidal doses with variable slope models were used with the following equation:

$$
y=\min +\frac{\max -\min }{1+10^{\left(\left\{\log I C_{50}-x\right\} \times \text { Hill slope }\right)}}
$$

where $y$ is the absorbance at $405 / 750 \mathrm{~nm}$; $x$ is the logarithm of the drug concentration, min is the absorbance at $405 / 750 \mathrm{~nm}$ measured at time zero (starting point of assay), and max is the maximal absorbance of a particular drug. The Hill slope is the steepness of the curve. The logarithm of the concentration at zero was defined at $2 \log$ lower than the lowest concentration of a particular drug.

\section{Results and discussion}

Relationship between the absorbance of the haem content of haemozoin and parasitaemia

The relationship between the haemozoin amount in a parasitized erythrocyte and parasitaemia was revealed by measuring the absorbance of the haem content of the haemozoin obtained from the parasitized erythrocytes after degradation to monomer haem. As shown in Figure 1, the absorption of converted haem showed a direct and linear correlation with the level of parasitaemia. Low and unsynchronized parasitaemia (about 1\%) could be detected using this haemozoin-based colorimetric method. The results indicate that this novel assay of parasites is applicable for monitoring parasite growth and for screening new antimalarial compounds.

\section{Haemozoin-based colorimetric assay to determine the $I_{50}$ values}

The $\mathrm{IC}_{50}$ values of some anti-malarial drugs were determined with a dose-response experiment using a haemozoin-based colorimetric assay. The result showed that increasing the concentration of anti-malarial drugs

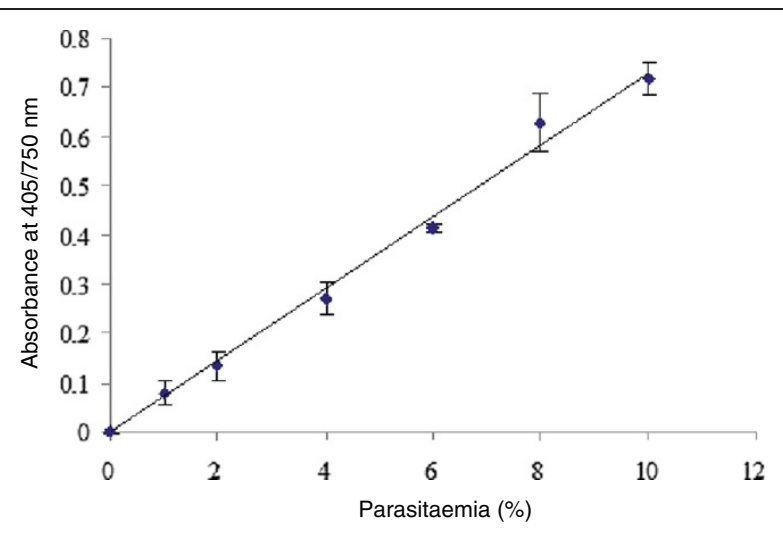

Figure 1 The linear relationship between the haemozoin level of a parasite and parasitaemia. Haemozoin concentration of infected erythrocytes is presented by the absorbance at 405/750 nm of monomeric haem after conversion from haemozoin using an $\mathrm{NaOH}$ solution. Absorption values (means \pm standard errors of triplicate wells) are plotted against parasitaemia. A well correlated, linear relationship $\left(R^{2}=0.9929\right)$ is strong evidence of the sensitivity of the method.

resulted in a decreased absorbance at 405/750 nm (Figure 2). The data was best fitted by a typical sigmoidal doseresponse model with a variable slope (four parameters) that agreed well with previous reports [26,27].

Table 1 summarizes the results of the assay to determine $\mathrm{IC}_{50}$ using the haemozoin-based colorimetric method. The $P$. falciparum $\mathrm{K} 1$ strain was primarily observed for quinine, chloroquine, clotrimazole, pyrimethamine and artemisinin, with $\mathrm{IC}_{50}$ values of 0.258 ,

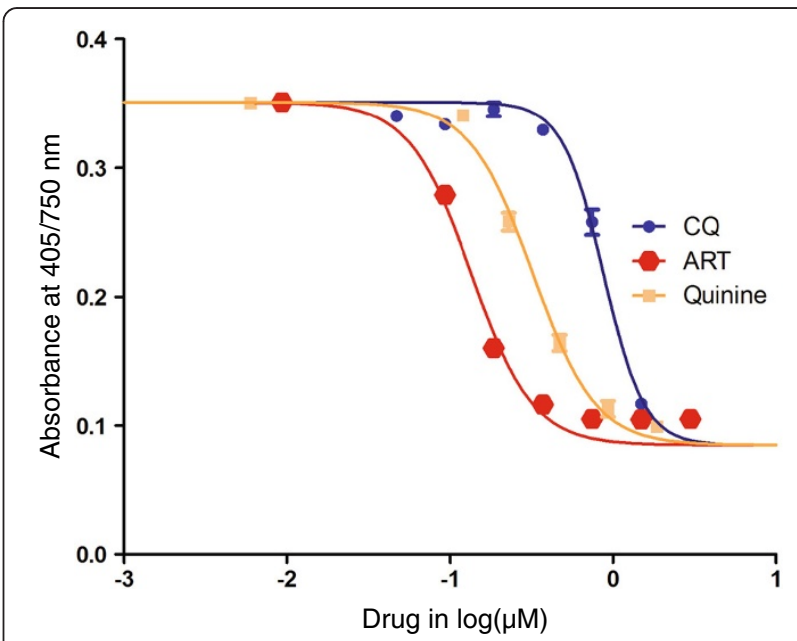

Figure 2 Representative dose responses for chloroquine (CQ), artemisinin (ART) and quinine against the Plasmodium falciparum K1 strain. Parasite growth after incubation of parasitized erythrocytes for $72 \mathrm{hr}$ with a drug was measured using a haemozoin-based colorimetric method. The symbols and error bars are the average absorption values at 405/750 $\mathrm{nm}$ and standard deviations in triplicate, respectively. The sigmoidal dose response model with a variable slope is the best fit to the data. 
Table 1 In vitro anti-malarial activities of drugs against chloroquine-susceptible (9A) and -resistant (K1) strains of Plasmodium falciparum

\begin{tabular}{lll}
\hline Drugs & \multicolumn{2}{c}{ Mean $\mathrm{IC}_{\mathbf{5 0}}$ and $\mathbf{9 5 \%} \mathrm{Cl}(\boldsymbol{\mu M})$} \\
\cline { 2 - 3 } & $\boldsymbol{P . \text { falciparum } \mathrm { K } \text { 1 strain }}$ & $\boldsymbol{P}$. falciparum 9A strain \\
\hline Quinine & $0.258(0.242-0.275)$ & $0.398(0.307-0.516)$ \\
Chloroquine & $0.873(0.824-0.926)$ & $0.132(0.082-0.211)$ \\
Clotrimazole & $0.805(0.689-0.939)$ & $1.67(1.18-2.37)$ \\
Pyrimethamine & $23.03(18.36-28.90)$ & Not done \\
Artemisinin & $0.139(0.124-0.155)$ & $0.483(0.376-0.622)$ \\
\hline
\end{tabular}

Parasitized red blood cells were incubated with different concentrations of drugs for $72 \mathrm{hr}$ and parasite growth was evaluated using the haemozoinbased colorimetric method. The IC $\mathrm{C}_{50}$ and its $95 \%$ confidence interval $(95 \% \mathrm{Cl})$ were calculated from the concentration-response curve of the haemozoin level vs the log concentration of a drug.

$0.873,0.805,23.03$, and $0.139 \mu \mathrm{M}$, respectively. For the $P$. falciparum 9A strain, pyrimethamine was not evaluated, and the $\mathrm{IC}_{50}$ values were $0.398,0.132,1.67$ and $0.483 \mu \mathrm{M}$ in succession for quinine, chloroquine, clotrimazole and artemisinin, respectively. The $\mathrm{IC}_{50}$ values for quinine, chloroquine, clotrimazole, and pyrimethamine were in a range that was similar to those observed in previous reports $[28,29]$. On the other hand, the $\mathrm{IC}_{50}$ values for artemisinine were higher than previous reports, probably due to asynchronous cultures, ring stage-specific target of artemisinine, accumulation of released hemozoin in the continuous cultures, or several rounds of continuous cultures and cloning of parasite strains in our laboratories. Therefore, further studies are required to compare the novel assay with recent developed methods to validate the accuracy in the screening new antimalarial compounds [30]. Another limitation of the novel method is that it is not easily adaptable for a high throughput screening of anti-malarial drug candidates, which is under-developed using 96-well filter plates [31].

In recent years, the number of laboratories, diagnosis centres and research institutes has risen in developing countries. However, most of them lack the modern equipment and expensive chemicals to apply new methods for screening anti-malarial candidates. In addition, some methods have potential risks of toxicity, so it is prudent to wear disposable gloves at all times when proceeding. Another obstacle is that many laboratories lack the facilities to treat toxic contamination before the toxin is discarded in the environment. The novel antimalarial assay is safe, non-expensive and easy to apply in laboratories.

\section{Conclusions}

The standard curve obtained in this study was strongly linear between the absorbance of monomeric haem converted from haemozoin and the percentage of parasitaemia. The $\mathrm{IC}_{50}$ values of chloroquine and quinine obtained from the haemozoin-based colorimetric method are similar to other methods. Even though this report describes specific conditions, the current experiment has introduced an assay that is adaptable to a wide range of conditions. The results also show that using this method has several advantages over using current methods. First, the method is fast and is based on a simple technique that uses a microplate reader, which is available in most laboratories. Second, the assay is based on inexpensive chemicals with no requirement of cold storage. Last but not least, the assay of the inhibition of $P$. falciparum growth using a haemozoin-based colorimetric method is feasible, reproducible, non-toxic, and more convenient than other assays, which makes it particularly useful for developing countries in the screening of novel anti-plasmodials, as a useful high throughput screening method for anti-malarial drug candidates.

\section{Competing interests}

The authors declare that they have no competing interests.

\section{Authors' contributions}

NTH and KK developed the idea for the project. TTM and NTH conceived and designed the experiments. TTM, NTH and MNS carried out the laboratory work. TTM, NTH, DTXT, KH, and KK analysed and interpreted the data. TTM, NTH, MNS, KH and KK contributed reagents/materials/analysis tools. . TTM, NTH, DTXT and KK wrote the paper. All authors had full access to all data in the study, read and approved the manuscript.

\section{Acknowledgements}

We gratefully acknowledge the Japan Student Services Organization (JASSO) that provided a scholarship for a short-time study in Japan (student exchange support programme) to Tran Thanh Men. This study was supported in part by the Global COE Program (2008 - 2012) and the Japan Initiative for Global Research Network on Infectious Diseases (J-GRID) for $\mathrm{KH}$.

\section{Author details}

'Department of Biology, College of Natural Science, Cantho University, Cantho City, Vietnam. ${ }^{2}$ Department of Biomolecular Engineering, Kyoto Institute of Technology, Matsugasaki, Sakyo-ku, Kyoto 606-8585, Japan. ${ }^{3}$ Department of Immunogenetics, Institute of Tropical Medicine (NEKKEN), Nagasaki University, Nagasaki, Japan. ${ }^{4}$ Global COE program, Nagasaki University, Nagasaki, Japan.

Received: 8 May 2012 Accepted: 13 July 2012

Published: 9 August 2012

\section{References}

1. Breman JG, Alilio MS, Mills A: Conquering the intolerable burden of malaria: what's new, what's needed: a summary. AmJTrop Med Hyg 2004 71:1-15.

2. Wongsrichanalai $C$, Pickard $A L$, Wernsdorfer WH, Meshnick SR: Epidemiology of drug-resistant malaria. Lancet Infect Dis 2002, 2:209-218.

3. Adjuik M, Babiker A, Garner P, Olliaro P, Taylor W, White N: Artesunate combinations for treatment of malaria: meta-analysis. Lancet 2004, 363:9-17.

4. WHO: Guidelines for the treatment of malaria. Geneva, Switzerland: World Health Organization; 2006.

5. Muller $\mathrm{O}$, Sie A, Meissner $\mathrm{P}$, Schirmer $\mathrm{RH}$, Kouyate B: Artemisinin resistance on the Thai-Cambodian border. Lancet 2009, 374:1419.

6. Dondorp AM, Nosten F, Yi P, Das D, Phyo AP, Tarning J, Lwin KM, Ariey F Hanpithakpong W, Lee SJ, Ringwald P, Silamut K, Imwong M, Chotivanich K, Lim P, Herdman T, An SS, Yeung S, Singhasivanon P, Day NP, Lindegardh N, Socheat $\mathrm{D}$, White $\mathrm{NJ}$ : Artemisinin resistance in Plasmodium falciparum malaria. N Engl J Med 2009, 361:455-467. 
7. Noedl H, Se Y, Sriwichai S, Schaecher K, Teja-Isavadharm P, Smith B, Rutvisuttinunt W, Bethell D, Surasri S, Fukuda MM, Socheat D, Chan Thap L: Artemisinin resistance in Cambodia: a clinical trial designed to address an emerging problem in Southeast Asia. Clin Infect Dis 2010, 51:e82-e89.

8. Desjardins RE, Canfield CJ, Haynes JD, Chulay JD: Quantitative assessment of antimalarial activity in vitro by a semiautomated microdilution technique. Antimicrob Agents Chemother 1979, 16:710-718.

9. Elabbadi $\mathrm{N}$, Ancelin ML, Vial HJ: Use of radioactive ethanolamine incorporation into phospholipids to assess in vitro antimalarial activity by the semiautomated microdilution technique. Antimicrob Agents Chemother 1992, 36:50-55

10. Corbett Y, Herrera L, Gonzalez J, Cubilla L, Capson TL, Coley PD, Kursar TA, Romero LI, Ortega-Barria E: A novel DNA-based microfluorimetric method to evaluate antimalarial drug activity. AmJTrop Med Hyg 2004, 70:119-124.

11. Quashie NB, de Koning HP, Ranford-Cartwright LC: An improved and highly sensitive microfluorimetric method for assessing susceptibility of Plasmodium falciparum to antimalarial drugs in vitro. Malar J 2006, 5:95.

12. Smilkstein M, Sriwilaijaroen N, Kelly JX, Wilairat $P$, Riscoe M: Simple and inexpensive fluorescence-based technique for high-throughput antimalarial drug screening. Antimicrob Agents Chemother 2004, 48:1803-1806.

13. Vossen MG, Pferschy S, Chiba P, Noedl H: The SYBR Green I malaria drug sensitivity assay: performance in low parasitemia samples. AmJTrop Med Hyg 2010, 82:398-401.

14. Noedl H, Wernsdorfer WH, Miller RS, Wongsrichanalai C: Histidine-rich protein II: a novel approach to malaria drug sensitivity testing. Antimicrob Agents Chemother 2002, 46:1658-1664.

15. Makler MT, Ries JM, Williams JA, Bancroft JE, Piper RC, Gibbins BL, Hinrichs DJ: Parasite lactate dehydrogenase as an assay for Plasmodium falciparum drug sensitivity. AmJTrop Med Hyg 1993, 48:739-741.

16. Druilhe P, Moreno A, Blanc C, Brasseur PH, Jacquier P: A colorimetric in vitro drug sensitivity assay for Plasmodium falciparum based on a highly sensitive double-site lactate dehydrogenase antigen-capture enzyme-linked immunosorbent assay. AmJTrop Med Hyg 2001, 64:233-241.

17. Eckman JR, Modler S, Eaton JW, Berger E, Engel RR: Host heme catabolism in drug-sensitive and drug-resistant malaria. J Lab Clin Med 1977, 90:767-770.

18. Huy NT, Serada S, Trang DT, Takano R, Kondo Y, Kanaori K, Tajima K, Hara S, Kamei K: Neutralization of toxic heme by Plasmodium falciparum histidine-rich protein 2. J Biochem 2003, 133:693-698.

19. Sullivan DJ Jr, Gluzman IY, Goldberg DE: Plasmodium hemozoin formation mediated by histidine-rich proteins. Science 1996, 271:219-222.

20. Atamna $H$, Ginsburg $H$ : Heme degradation in the presence of glutathione. A proposed mechanism to account for the high levels of non-heme iron found in the membranes of hemoglobinopathic red blood cells. J Biol Chem 1995, 270:24876-24883.

21. Huy NT, Kamei K, Yamamoto T, Kondo Y, Kanaori K, Takano R, Tajima K, Hara $S$ : Clotrimazole binds to heme and enhances heme-dependent hemolysis: proposed antimalarial mechanism of clotrimazole. J Biol Chem 2002, 277:4152-4158.

22. Francis SE, Sullivan DJ Jr, Goldberg DE: Hemoglobin metabolism in the malaria parasite Plasmodium falciparum. Annu Rev Microbiol 1997, 51:97-123.

23. Maeno Y, Nakazawa S, le Dao D, Yamamoto N, Giang ND, Van Hanh T, le Thuan K, Taniguchi K: A dried blood sample on filter paper is suitable for detecting Plasmodium falciparum gametocytes by reverse transcription polymerase chain reaction. Acta Trop 2008, 107:121-127.

24. Trager $W$, Jensen JB: Human malaria parasites in continuous culture. Science 1976, 193:673-675.

25. Takeuchi Y, Yamakawa N, Kaetsu M, Fujiwara K, Okada J: Experiences with AnaeroPack systems. J Jpn Assoc Anaerob Infect Res 1992, 22:106-112.

26. Shuaibu MN, Wuyep PA, Yanagi T, Hirayama K, Tanaka T, Kouno I: The use of microfluorometric method for activity-guided isolation of antiplasmodial compound from plant extracts. Parasitol Res 2008, 102:1119-1127.

27. Bhattacharya A, Mishra LC, Bhasin VK: In vitro activity of artemisinin in combination with clotrimazole or heat-treated amphotericin $B$ against Plasmodium falciparum. AmJTrop Med Hyg 2008, 78:721-728.
28. Karl S, Wong RP, St Pierre TG, Davis TM: A comparative study of a flowcytometry-based assessment of in vitro Plasmodium falciparum drug sensitivity. Malar J 2009, 8:294.

29. Kurosawa Y, Dorn A, Kitsuji-Shirane M, Shimada H, Satoh T, Matile H, Hofheinz W, Masciadri R, Kansy M, Ridley RG: Hematin polymerization assay as a high-throughput screen for identification of new antimalarial pharmacophores. Antimicrob Agents Chemother 2000, 44:2638-2644.

30. Franke-Fayard B, Djokovic D, Dooren MW, Ramesar J, Waters AP, Falade MO, Kranendonk M, Martinelli A, Cravo P, Janse CJ: Simple and sensitive antimalarial drug screening in vitro and in vivo using transgenic luciferase expressing Plasmodium berghei parasites. Int J Parasitol 2008, 38:1651-1662.

31. Baniecki ML, Wirth DF, Clardy J: High-throughput Plasmodium falciparum growth assay for malaria drug discovery. Antimicrob Agents Chemother 2007, 51:716-723.

doi:10.1186/1475-2875-11-272

Cite this article as: Men et al:: A simple and inexpensive haemozoinbased colorimetric method to evaluate anti-malarial drug activity.

Malaria Journal 2012 11:272.

\section{Submit your next manuscript to BioMed Central and take full advantage of:}

- Convenient online submission

- Thorough peer review

- No space constraints or color figure charges

- Immediate publication on acceptance

- Inclusion in PubMed, CAS, Scopus and Google Scholar

- Research which is freely available for redistribution

Submit your manuscript at www.biomedcentral.com/submit
C) Biomed Central 